\title{
Stage Identification and Classification of Lung Cancer using Deep Convolutional Neural Network
}

\author{
Varsha Prakash ${ }^{1}$, Smitha Vas.P ${ }^{2}$ \\ Dept. of Computer Science and Engineering \\ LBS Institute of Technology for Women \\ Trivandrum, India
}

\begin{abstract}
The performance of lung segmentation is highly dependent on disease prediction task. Challenges for prediction and segmentation raise the need of using multiple learning techniques. Current models initially perform image segmentation in all CT scan images and then classify it as malicious or benign. This consumes more time since it segments both normal and abnormal CT's. So, due to improper segmentation of images the region of interest will be inaccurate and results in false classification of images. Therefore, by initially checking the CT which has malignancy and then segmenting those lesions will provide more accuracy in segmentation of cancerous nodules thereby helps to identify the stage of cancer the patient is suffering from. The aim is to improve the current cancer detection techniques using DCNN by filtering out malignant CT scan from the medical dataset and segmenting those images for stage identification. Segmentation is done using UNET++ architecture and stage identification is done by considering the "size" (T) parameter from the globally recognized standard named "TNM staging" for classifying the spread of each malignant nodule as T1-T4. $99.83 \%$ accuracy is achieved in lung cancer classification using VGG-16 which yields better results for both segmentation and stage identification too.
\end{abstract}

Keywords-Computer Aided Diagnosis (CAD); Deep Convolutional Neural Network (DCNN); pulmonary nodule; segmentation; benign; malignant; staging

\section{INTRODUCTION}

Computed Tomography (CT) scans are well set up for detecting lung nodules [26]. A tumor is a mass of tissue that's formed by an accumulation of abnormal cells. Tumors can be benign or malignant. When we speak of "cancer" we refer to those tumors that are considered malignant. Malignant tumors that are fast growing and tend to spread to other parts of the body called metastasis, allowing tumor cells to enter the bloodstream or lymph system and other parts of the body, while benign tumors do not spread to other parts of the body. Lung cancer tends to spread at an early stage so, it is one of the most challenging tasks to diagnose the disease as early as possible. Especially the adrenal glands, liver, brain, and bone are some most prevalent places for lung cancer metastasis. But by using a single detector CT scan, the small lesions in the lung still remain difficult to spot. When pursuit early detection of lung cancer this poses itself as a dare. Since early detection is the key for a successful detection and healing, the incompetence to manually diagnose the small lesions further obstruct the possibility of early diagnosis.300 cross-sectional 2D images are currently produced by each CT scanners. And under particular time constraint each of which must be individually evaluated. Apart from the particular diagnostic benefits furnished by the CT imaging, the probability of error rate of cancer detection will be high due to the manual interpretation that is required for analyzing 200-300 slices per exam. Thus, detection of lung cancer becomes highly laborintensive since tumor vary in position, size, shape depending on the patient. This increases false positives in detecting cancer either by detecting cancer in a patient who is cancer free, or by failing to detect the tumor. Current studies of a radiologist who have examined more than $20 \mathrm{CT}$ examinations per day give evidence that $7 \%-15 \%$ is the rate of mispredictions occurring in CT based malignancy diagnosis systems. So, in order to tackle this issue CAD systems were nowadays used for medical diagnosis due to accessibility of surplus data pertaining to the CT scan patients. Several approaches like image processing, data mining, and machine learning [25] are proposed for addressing the disease prediction task. On that account, to verify that oddity and divergence properly handled models came into existence and for classification of complex images deep learning has come into existence. The performance of lung segmentation is highly dependent on disease prediction task. Challenges for prediction and segmentation raise the need of using multiple learning techniques.

Current models initially perform image segmentation in all CT scan images and then classify it as malicious or benign. This consumes more time since it segments both normal and abnormal CT's. So, due to improper segmentation of images the region of interest will be inaccurate and results in false classification of images. Therefore, by first checking the CT that has malignancy and then segmenting those lesions, it will provide more precision in the segmentation of cancerous nodules, thereby helping to identify the stage of cancer that the patient is suffering from. Cancer detection technique is improved using DCNN by filtering out malignant CT scan from whole medical dataset and segmenting those images using UNET++ for stage identification and stage identification is done by considering the "size" i.e. T parameter from the standard named "TNM staging”.

The paper is organized as Section II, discusses major detection techniques done in lung cancer. Section III explains the proposed approach comprising of three phases viz. Lung Cancer Detection, Malignant Nodule Segmentation, Lung Cancer Stage Identification respectively. Section IV discusses the details of the implementation and dataset used. Section V 
deals with the experimental findings and ultimately Section VI deals with the conclusion.

\section{LITERATURE SURVEY}

In the literature number of researches on image processing techniques exist to detect cancer detection. But there is no improvement in the hit ratio of early stage cancer detection. With the advancement in machine learning techniques, many researchers are trying to diagnose cancer early as possible. Neural network plays a major part in recognizing cancerous area(malignant), which provides an effective tool for cancer detection. The cancer treatment will only be effective if the tumor cells are precisely separated from the normal cells. For each medical recognition task, differently modelled CNN needs to be employed. Compared to conventional methods, deep convolutional neural network gives much more processing magnitude and it gives more explicit results.

A Discriminative Feature Network (DFN), was introduced in [1] which contains Smooth Network and Border Network. A Smooth Network was introduced, which contains both Channel Attention Block and global average pooling to point out the discriminative features. A Border Network was also introduced along with it to distinguish boundaries of bilateral features with deep semantic boundary supervision to handle the intra-class in-consistency problem. Smaller strides or patching with a lot of overlapping in done in both which results in computationally intensive results in redundant information. Secondly, in the field of biomedical image, analysis is constrained by the lack of huge annotated samples. Small patches and large patches result in loss of contextual information and tamper with the localization results respectively. Loss of context information appears due to nonoverlapping patches. An architecture called U-Net convolution is implemented in [2] exclusively for segmenting the biomedical images. Primarily U-Net employs data augmentation technique which does not create limitation in the number of data. Different color contrast remains to be a challenging task due to the staining procedure, cell structure in various histopathological images. So, in [3] not only extract the semantic information of the former layer, this do pay much attention to the shallower layers' semantic information by introducing RIC-UNET for image segmentation and classification. Residual block extract more characteristic features for segmentation. Inception module is for its computational efficiency while incorporating multiscale features with kernels of different sizes. Channel attention mechanism can focus the parameter training on the region of interest and alleviate the over fitting problem. Whole mammogram image was trained to an End to end deep multiinstance networks for mass classification. It was not on the basis of region of interest (ROI). So, in [4] introduced CAD systems for mammogram samples. It classifies both benign and malignant mass lesions. Using a threshold and regionbased techniques for segmentation, DCNN is connected to SVM to obtain better classification results. Particle Swarm Optimization (PSO) algorithm based 2-D CNN is discussed in [5] using LIDC dataset to remove the network hyperparameter's manual searching. 2-D U-Net is employed along with an ensemble of XGBoost and RF for nodule feature classification in [6]. Employing 3-DCNN, pulmonary nodule detection can be done through reinforcement learning technique. In [7] proposed a 3-dimensional U-Net, Dense Net and Region Proposal Network for pulmonary nodule detection in CT lung images. Here, candidate selection is done through U-net based RPN and false classification is reduced by using Dense Net. In [8] 3-D faster RCNN model similar to U-Net is used for creating nodule and DCNN is employed for finding out nodules.3-D based group convolutional neural network (G-CNN) [9] is proposed for medical diagnosis having almost similar performance rate as that of normal CNN but demands high utility of data than normal CNN. Various-sized input data for classification of lung nodule as benign and malicious are introduced by incorporating multiple 3-D CNN [10]. Prediction can also render both 2-D and 3-D approaches. In 2D CNN [11], both local and global features are taken and classified. The hybrid version of Alex Net and LeNet determines the malignancy in the tumor by just adopting the layers from LeNet and network parameters from Alex Net thereafter it got its name as Agile CNN[12] and have an accuracy of $82.2 \%$.For finding the degree of malignancy in lung images, 3-D CNN model uses different approaches mainly by adopting previous multi-task learning techniques along with width and center of windows [13] and fusing both 3-D dual path Conventional Neural Network and GBM [14].Using a noisy U-Net [15] improved neural network sensitivity and small nodule detection enhancement by creating a special noise for training in the hidden layer lung nodule identification can be done using various morphological features by training two models both RCNN and CNN to predict lung nodules in CT scan images [16]. Pre-processing CT scan images are done through blurring and thresholding so as to enhance the quality of the input and through splitting left and right lung to reduce the complexity the image and to increase accuracy in DCNN, is defined in [17]. Four different sized 3D CNN [18] also provide higher accuracy for detecting the lesions irrespective of their size.

\section{PROPOSED METHOD}

The proposed methodology employs three stages: Lung Cancer detection, segmentation and stage identification respectively. The first one is the lung cancer detection which will classify the lung CT images into benign or malicious and thus from the dataset abnormal lung CT can be extracted. The second component is malignant nodule segmentation which is used for extracting nodule portions from the malicious lung. The third component is lung cancer stage identification which will find out the stages of all nodes that have been segmented using UNet++ based on popular cancer staging standard named TNM staging which was developed by Union of International Cancer Control (UICC). Based on the size of the nodules(T), it is classified into different stages which will helps to diagnose patient's degree of spread of disease more accurately by an oncologist than previous existing methods. Overview of proposed method is shown in Fig. 1.

\section{A. Lung Cancer Detection}

Lung Cancer Detection is done using VGG-16 CNN which is shown in Fig. 2. 


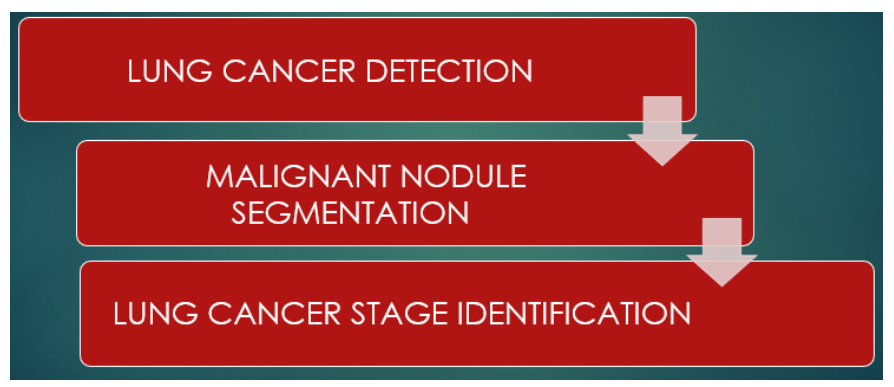

Fig. 1. Proposed Modules.

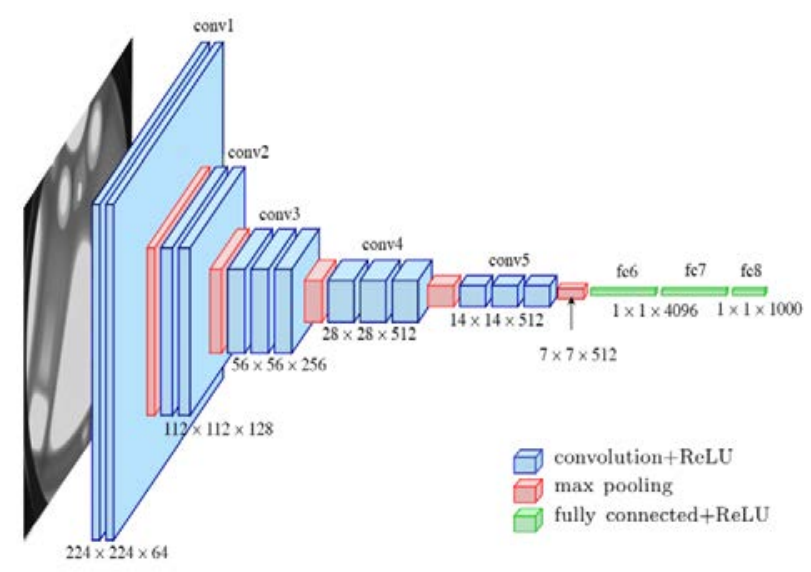

Fig. 2. VGG-16 Architecture (Source: [27]).

DCNN is used to detect lung cancer based on chest CT images [21]. Lung regions are extracted from CT images in the first stage. It is used to train the medical images and thus helps to diagnose the patient's disease. The main objective is to detect whether the CT of the lung is malignant or benign [23]. Here, VGG-16 is used for classification. In VGG architecture [20], filters are used by all convolutional layers with small receptive field of size $3 \times 3$. Convolutional layers are followed by max spatial pooling over $2 \times 2$ window and stride 2 which effectively down samples resolution by a factor 2 . Two fully connected layers, each having 4096 channels, and layer with 2 sigmoid activated outputs corresponding to benign and malicious class are connected to the last pooling layer.

\section{B. Malignant Nodule Segmentation}

For nodule segmentation, U-net++ [22] architecture is used. Input for segmentation would be the malignant images that we got from previous module. Those images and its mask are generated and both are given as input for training the model. Training data contains 30 images which are not satisfactory to cater a deep learning neural network. So, data augmentation is done to increase the input samples for better training. Output obtained from the network represent mask from which learning should be done. The mask pixels should be in $[0,1]$ range which is ensured by sigmoidal activation functions. In U-net++ architecture there is two parts namely the expansive and contraction path in which it poses series of nested convolution block to turn down the semantic gap in both encoder-decoder parts. Along with that, it can skip pathways which enable to prune network according to our requirements at any time. Architecture of $\mathrm{UNet}^{++}$is shown in Fig. 3.

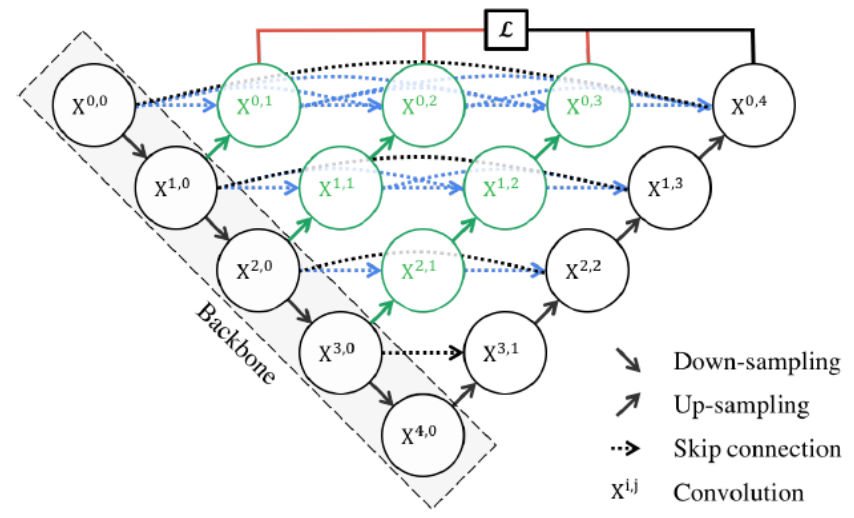

Fig. 3. UNet++ Architecture (Source: [28]).

In contracting path, each task account for two convolutional layers. The number of channels vary from $1 \rightarrow$ 64 as convolution rises the depth of the image. The max pooling process halves down the image size owing to padding issues. But here padding= "same" and the process is repeated for 3 more times. 2 convolutional layers are built at the bottom part with no max pooling and as a result image has been resized. In the expansive path, image is upsized to its native size by using Transposed convolution. Padding operation is applied on the original image go around with a convolution operation. The image is upsized after transposed convolution and this image is merged with the corresponding image produced from the contracting path. More precise prediction can be achieved by combining information from the previous layers. Again, two other convolution layers are included. This process is repeated 3 more times. The last step is to reshape the image. The last layer is a convolution layer and having one filter with size $1 \times 1$.And the rest is same for neural network training. By giving any of the malicious input along with mask, testing is done which provide right segmented nodule. From the segmentation module, .png image will be generated as output.

\section{Lung Cancer Stage Identification}

Based on the area of malignant nodule, the stage of cancer can be identified. It will show how big the disease is, and will also help to find out whether it has spread to another part of our body. Stage identification is very important since it helps the doctor to diagnose the cancer into limited or extended. In order to accomplish this, TNM staging, a globally recognized standard is used to determine the spread of lung cancer. In TNM staging, three parameters are used to determine how severely the patient is affected. Parameters are $\mathrm{T}$ to denote size of the tumor; $\mathrm{N}$ to denote whether it has spread to lymph nodes and $\mathrm{M}$ denotes metastasis which indicates whether cancer have spread to different region or not. As for now, in this work only tumor size is taken into consideration with labels T1 to T4.

\section{IMPLEMENTATION}

The trained system can detect presence of cancer in CT image of the lung and only the malignant image is given to UNet++ for segmentation. From the segmented image stage identification is done i.e., stage labels like T1, T2, T3, T4. Fig. 4 shows the block diagram of the proposed system. 


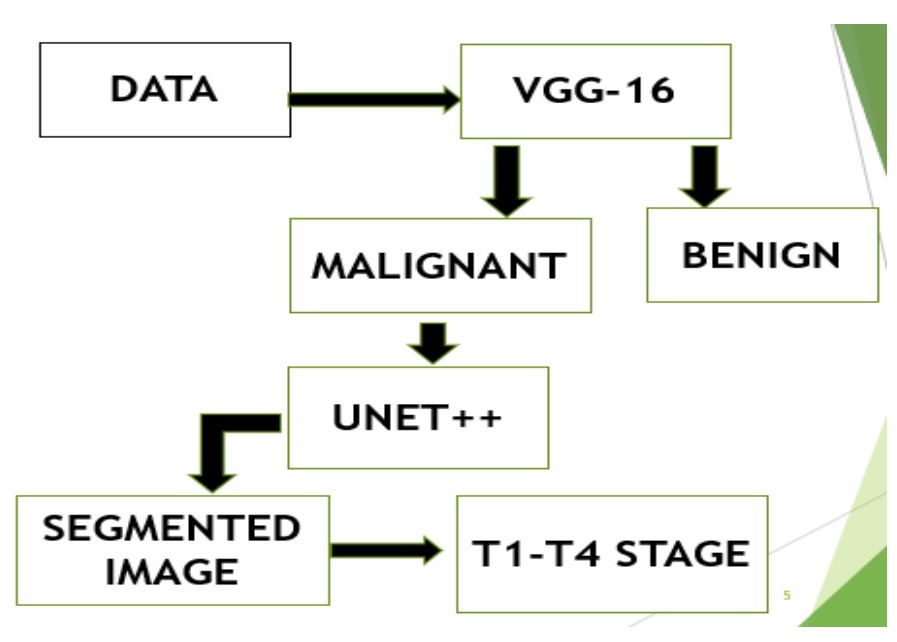

Fig. 4. Block Diagram.

Codes are executed in Google Collaboratory. This research tool is a Jupyter notebook environment runs on dedicated servers generally used for machine learning works. 2-core Xeon 2.2GHz processor, 33GB space, 13GB system memory and an NVDIA Tesla K80gpu are the features of Colab VM. Here, Keras on the TensorFlow background is used to run the code. Once the images are ready in its binary matrix format, dataset is split into three sets as training, validation and testing phase within the ratio 70\%:15\%:15\%. In general, DNN requires large data set for train-testing, so data augmentation [19] is applied in order to obtain larger data set. For that various augmentation techniques like rotation, zoom, shear, flipping is done on the same images which can be used in the training process, and thus training algorithm can use a larger data set created at train time. Elements that have below 50\% of success rate will be dropped out by Learning rate and the dropout factor. Dataset (X, Y) will be taken in training process and data set (X_Test, Y_Test) for testing. The algorithm passes all the images in every train cycle. Our training process has 100 epochs to train 200 large scale CT images with batch size 128. After the completion of training, the network is saved and can be used to separate CT images into benign or malignant. As activation function for the output layer, SoftMax is used and cross entropy as loss functions. In testing a single image or a group of images were fed to the trained model. For predictions, path will be for single image testing and for group testing limit is given.

Malicious lung along with its corresponding masks are taken as input for segmentation with its dimensions resized from $224 * 224$ to $128 * 128$. Training is done using U-NET++ architecture along with their corresponding mask and it may take between 10 to 20 minutes depending upon the systems performance. Training step can be 5 epoch which will be fair for configuration. And once the training is completed, model is created. For training, weight optimizer Adam and sigmoid activation function is used with batch size equals 32. For each convolution layer, kernel size is $3 \mathrm{X} 3$ and with a stride of 2 . After each convolutional layer, required padding is added to keep the image size same. Overfitting in deep neural network will be avoided by using dropout factor. After segmentation of image, morphological operation is used to get the lung contour and to eliminate unnecessary parts. By doing morphological operations, it gets not only the lung but also apparent lung nodule. And then using the segmented nodule, nodule area is calculated. For that, according to the TNM staging standard for cancer detection, there is a particular threshold for labelling each nodule in different stages. The stages are from T1 to T4 and this will show how progressively cancer is affecting the lung. Each nodule stages size limit is shown in Table I.

TABLE I. Threshold VALUes of Lung CANCER NOdULES Based ON SIZE IN TNM STAGING STANDARDS

\begin{tabular}{|l|l|l|}
\hline STAGING & SUB-STAGING & NODULE SIZE \\
\hline \multirow{3}{*}{ T1 } & T1(a) & Between $3 \mathrm{~mm}$ and $10 \mathrm{~mm}$ \\
\cline { 2 - 3 } & T1(b) & Between $10 \mathrm{~mm}$ and $20 \mathrm{~mm}$ \\
\cline { 2 - 3 } & T1(c) & Between $20 \mathrm{~mm}$ and $30 \mathrm{~mm}$ \\
\hline \multirow{2}{*}{ T2 } & T2(a) & Between $30 \mathrm{~mm}$ and $40 \mathrm{~mm}$ \\
\cline { 2 - 3 } & T2(b) & Between $40 \mathrm{~mm}$ and $50 \mathrm{~mm}$ \\
\hline T3 & No Staging & Between $50 \mathrm{~mm}$ and 70 \\
\hline T4 & No Staging & Greater than $70 \mathrm{~mm}$ \\
\hline
\end{tabular}

A. Dataset

The Lung Image Database Consortium and Image Database Resource Initiative (LIDC-IDRI) database, provide training dataset in DICOM format with common tags which include the patient's name, age, sex, occupation and DICOM Unique Identifiers (UIDs) to indicate the patient's identity in DICOM which is put it into code within an image header. But considering the patient's confidentiality, all information should be removed from the DICOM header when a DICOM file is uploaded for research purposes. So, an efficient method is by converting DICOM file into JPEG image format. Since JPEG format is small in size, have lossless compression, readable by all computer platforms and highly portable they are the best preferred format since images are transferred over the Web. LIDC and IDRI consists of CT scan lung images containing both normal as benign and abnormal as malignant [24].

\section{EXPERIMENTAL RESULTS}

Predictions are done from lung cancer detection module through training the model using VGG-16 with given test images to display the correct labels as benign or malignant. Validation is also done to know how well this model is adapting to unseen data. Each batch, training will calculate loss with target output and expected output. Then calculate gradient of loss with respect to the model parameters. Estimation of val_loss, val_accuracy, train_loss, train_accuracy is done in plot.png corresponding to $20,40,60,80,100$ epoch. Validation loss and Training loss is gradually decreasing and Training accuracy and Validation accuracy is exponentially increasing and finally become stable which means the model built is learning and working fine. Fig. 5 shows the train_loss, val_acc, val_loss and train_acc obtained after training and validating the dataset. It yields $99.83 \%$ accuracy in classification. 


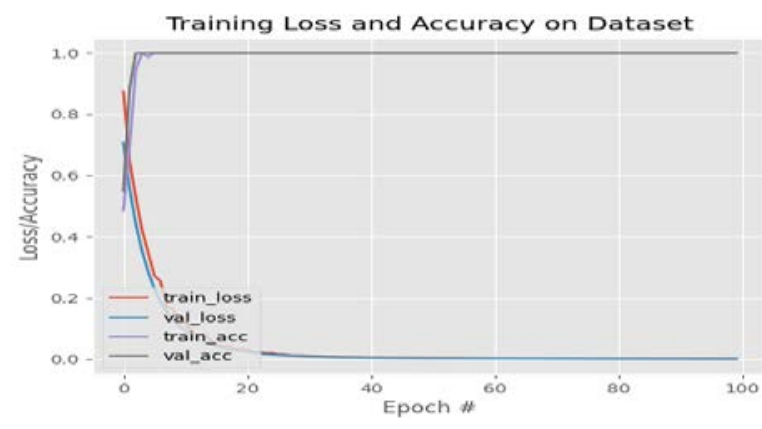

Fig. 5. Lung Cancer Prediction Result.

The result of lung cancer detection will be as benign or malignant based on the score values of each CT which is shown in Table II.

For nodule segmentation, input (malignant lung obtained after lung cancer detection module) and their corresponding mask will give the right segmentation result is shown in Fig. 6.

After segmentation contour along with pulmonary nodule is segmented using UNet++ which is shown in Fig. 7.

TABLE II. SCORE VAlues ObTAined AFTER Lung CANCER PREDiction

\begin{tabular}{|l|l|l|}
\hline LABELS & BENIGN & MALIGNANT \\
\hline NON-CANCEROUS & 0.998 & 0.001 \\
\hline CANCEROUS & 0.002 & 0.988 \\
\hline
\end{tabular}
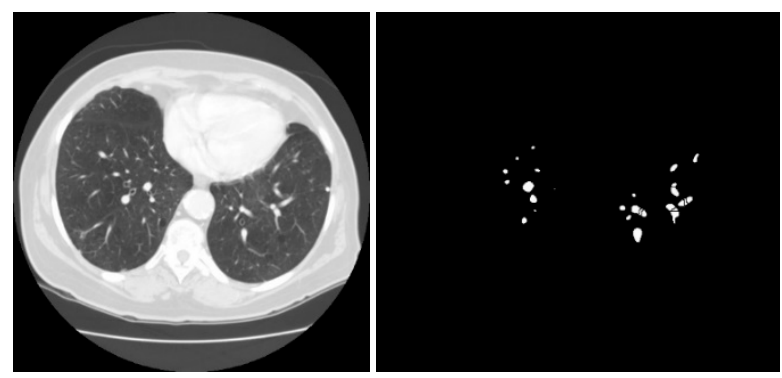

Fig. 6. Image and Corresponding Mask.

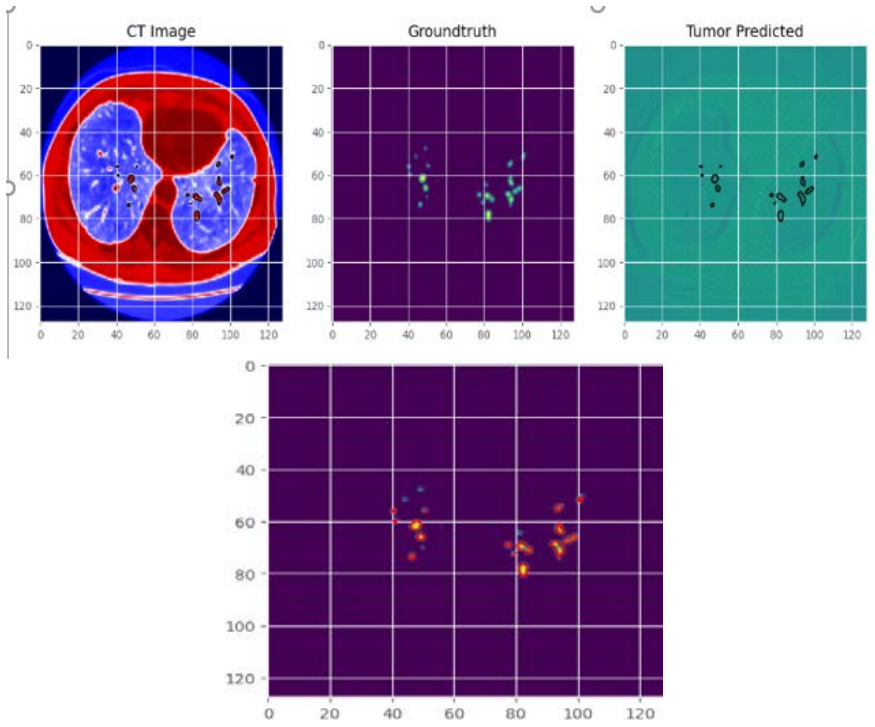

Fig. 7. Segmented Nodule.
After segmenting all nodules from malignant CT by UNet++, stages of segmented nodules are found by calculating the diameter of nodules present in the cancerous CT's. All nodules size between $3 \mathrm{~mm}$ and $10 \mathrm{~mm}$ is shown as red color in Fig. 8.

Here, two nodes are present in T1(a) stage. The corresponding nodules say nodule 1 and nodule 2's diameter is $4 \mathrm{~mm}$ and $5.5 \mathrm{~mm}$ respectively which is between $3 \mathrm{~mm}$ and 10mm. So, it lies in T1(a) Stage and is shown in Fig. 9.

Nodule's size between $10 \mathrm{~mm}$ and $20 \mathrm{~mm}$ is shown in red spot in Fig. 10.

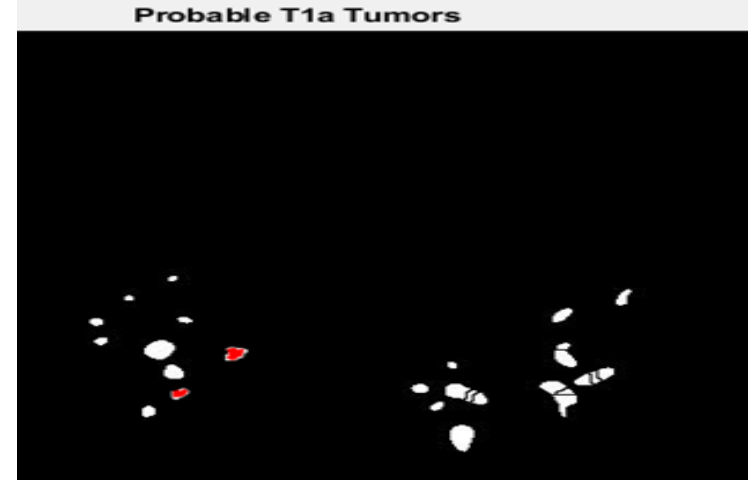

Fig. 8. Nodules Detected in T1(a) STAGE.

\section{Nodule \$2}
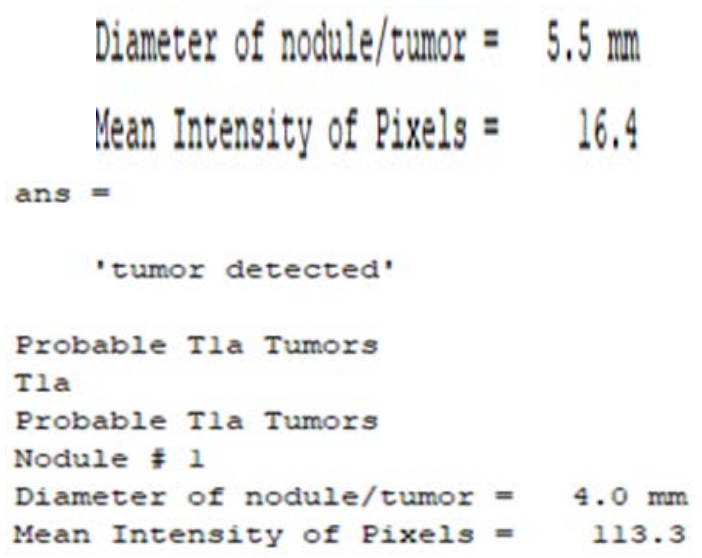

Fig. 9. Each Nodule in T1(a) STAGE.

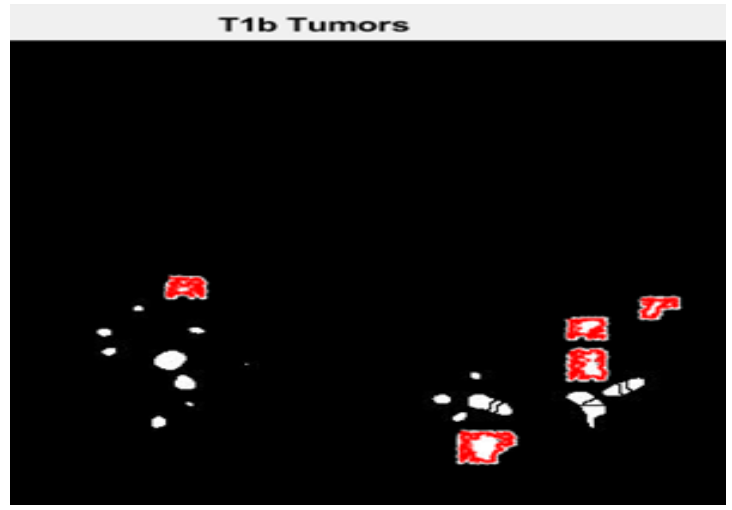

Fig. 10. Nodules Detected in T1(b) STAGE. 
Here, five nodes are present in T1(b) stage which is shown in the above result in red color. The corresponding nodules say nodule1, 2, 3, 4, 5's diameter is $10.8 \mathrm{~mm}, 15.3 \mathrm{~mm}$, $10.7 \mathrm{~mm}, 14.8 \mathrm{~mm}, 11.8 \mathrm{~mm}$ respectively which is between $10 \mathrm{~mm}$ and $20 \mathrm{~mm}$.So it lies in T1(b) Stage and each nodules size will be shown in Fig. 11.

Nodule's size between $20 \mathrm{~mm}$ and $30 \mathrm{~mm}$ is shown in green spot in Fig. 12.

\section{Frobable Tib Tumors \\ Tib

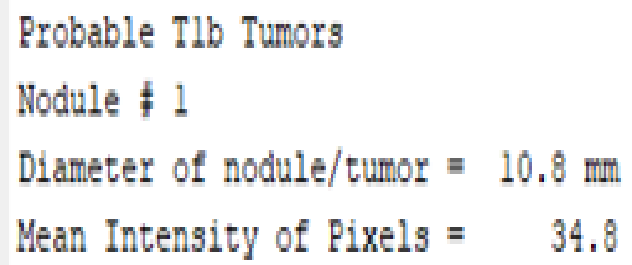

Here, one node is detected in $\mathrm{T} 1$ (c) with size $26.5 \mathrm{~mm}$ which is between $20 \mathrm{~mm}$ and $30 \mathrm{~mm}$ is shown in Fig. 13.

\section{Probable TlcTumors}

\section{Probable Tlc Tumors}

TlC

Nodule \# 1

Diameter of nodule/tumor $=26.5 \mathrm{~mm}$
Mean Intensity of Pixels $=180.6$

Fig. 13. Nodule in T1(c) STAGE.

Nodules size greater than $70 \mathrm{~mm}$ is shown in yellow spot in Fig. 14.

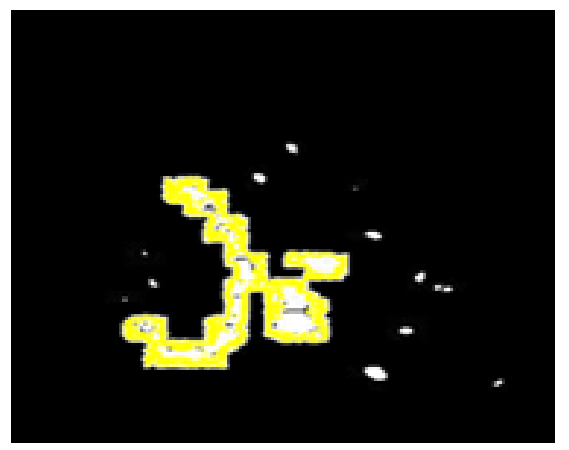

Fig. 14. Nodules Detected in T4 STAGE.

Here, one node is detected in T4 with size $73.9 \mathrm{~mm}$ which is greater than $70 \mathrm{~mm}$ is shown in Fig. 15.

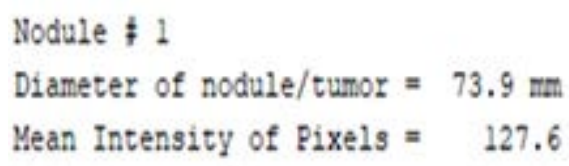

Fig. 15. Nodule in T4 STAGE.

\section{Nodule $\$ 5$}

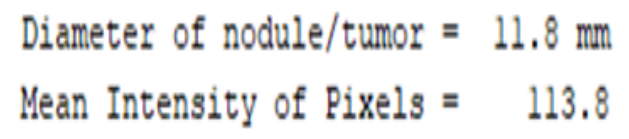

Fig. 11. Each Nodule in T1(b) STAGE.

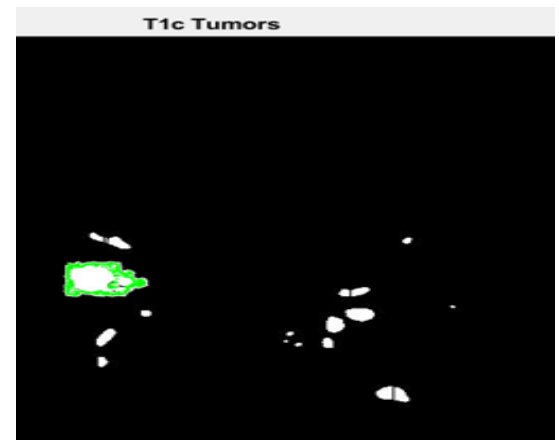

Fig. 12. Nodules Detected in T1(c) STAGE. 
a lot of automated systems are introduced to address the issue of reducing false positive while estimating the presence of pulmonary nodules in the CT scans of the patients. From here, we have the opportunity to use current topics to identify hidden patterns in nodules and construct a model to increase the probability of malignant tumor detection along with the identification of different stages of cancer. In future, parameters like whether malignancy have spread to other parts of the body(M) and whether it affects to the lymph nodes(N) can also be considered along with the tumor size(T) as parameters for lung cancer stage identification which will give much more accuracy in finding stages.

\section{ACKNOWLEDGMENT}

First and foremost, I express my deepest thanks to our Principal, Dr. Abdul Rahiman, for providing us with all facilities for the completion of this work. I am extremely happy to mention a great word of gratitude to Prof Smitha E.S, Professor and Head of the Department, Department of Computer Science and Engineering, for her aspiring guidance, invaluably constructive criticism and friendly advice during the work.

\section{REFERENCES}

[1] Yu, Changqian, Jingbo Wang, Chao Peng, Changxin Gao, Gang Yu, and Nong Sang, "Learning a discriminative feature network for semantic segmentation", In Proceedings of the IEEE conference on computer vision and pattern recognition, 2018.

[2] Shaziya H, Shyamala K and Zaheer R,” Automatic lung segmentation on thoracic ct scans using u-net convolutional network", In2018 International Conference on Communication and Signal Processing (ICCSP) 2018 April 3.

[3] Zeng, Zitao, Weihao Xie, Yunzhe Zhang, and Yao Lu,"RIC-Unet: An improved neural network based on Unet for nuclei segmentation in histology images", Ieee Access 7 (2019): 21420-21428.

[4] Ragab, Dina A., Maha Sharkas, Stephen Marshall, and Jinchang Ren, "Breast cancer detection using deep convolutional neural networks and support vector machines", PeerJ 7 (2019): e6201.

[5] G. L. F. da Silva, T. L. A. Valente, A. C. Silva, A. C. de Paiva, and M. Gattass, "Convolutional neural network-based PSO for lung nodule false positive reduction on CT images," Comput. Methods Programs Biomed., vol. 162, pp. 109-118, August. 2018.

[6] A. J. Jia, B. J. Liu, and C. Y. Gu, "Computer-aided diagnosis of pulmonary nodules on CT scan images," in Proc. 10th Int. Conf. Modelling, Identificat. Control (ICMIC), Guiyang, China, 2018, pp. 1-6.

[7] Y. Qin, H. Zheng, Y.-M. Zhu, and J. Yang, "Simultaneous accurate detection of pulmonary nodules and false positive reduction using 3D CNNs," in Proc. IEEE Int. Conf. Acoustic, Speech Signal Process. (ICASSP), Calgary, AB, Canada, April. 2018, pp. 1005-1009.

[8] H. Tang, D. R. Kim, and X. Xie, "Automated pulmonary nodule detection using 3D deep convolutional neural networks," in Proc. IEEE 15th Int. Symp. Biomed. Imag. (ISBI), Washington, DC, USA, April. 2018, pp. 523-526.

[9] M. Winkels and T. S. Cohen, "3D G-CNNs for pulmonary nodule detection,” 2018, arXiv:1804.04656. [Online]. Available: https://arxiv.org/abs/1804.04656

[10] P. Monkam, S. Qi, M. Xu, H. Li, F. Han, Y. Teng, and W. Qian, "Ensemble learning of multiple-view 3D-CNNs model for micronodules identification in CT images,” IEEE Access, vol. 7, pp. 55645576, 2018. doi: 10.1109/ACCESS.2018.2889350.

[11] W. J. Sori, J. Feng, and S. Liu, "Multi-path convolutional neural network for lung cancer detection," in Multidimensional Systems and Signal Pro- cessing. New York, NY, USA: Springer, 2018. doi: 10.1007/s11045-018- 0626-9.
[12] X. Zhao, L. Liu, S. Qi, Y. Teng, J. Li, and W. Qian, “Agile convolutional neural network for pulmonary nodule classification using CT images,"' Int.J. Comput. Assist. Radiol. Surg., vol. 13, no. 4, pp. 585-595, February. 2018.

[13] B. Wu, Z. Zhou, J. Wang, and Y. Wang, "Joint learning for pulmonary nodule segmentation, attributes and malignancy prediction," in Proc. IEEE 15th Int. Symp. Biomed. Imag. (ISBI), April 2018, pp. 1109-1113.

[14] W. Zhu, C. Liu, W. Fan, and X. Xie, "DeepLung: Deep 3D dual path nets for automated pulmonary nodule detection and classification," in Proc. IEEE Winter Conf. Appl. Comput. Vis. (WACV), March. 2018, pp. 673-681.

[15] Huang, Wenkai, and Lingkai Hu,"Using a Noisy U-Net for Detecting Lung Nodule Candidates", IEEE Access 7 (2019): 67905-67915.

[16] Wang, Weilun, and Goutam Chakraborty, "Deep Learning for Automatic Identification of Nodule Morphology Features and Prediction of Lung Cancer", 2019 IEEE 10th International Conference on Awareness Science and Technology (iCAST). IEEE, 2019.

[17] Rahman, Md Sakif, Pintu Chandra Shill, and Zarin Homayra, "A New Method for Lung Nodule Detection Using Deep Neural Networks for CT Images", 2019 International Conference on Electrical, Computer and Communication Engineering (ECCE). IEEE, 2019.

[18] Moradi, Pouria, and Mansour Jamzad, "Detecting Lung Cancer Lesions in CT Images using 3D Convolutional Neural Networks", 2019 4th International Conference on Pattern Recognition and Image Analysis (IPRIA). IEEE, 2019.

[19] Perez, Luis, and Jason Wang, "The effectiveness of data augmentation in image classification using deep learning", arXiv preprint arXiv:1712.04621 (2017).

[20] Simonyan, Karen, and Andrew Zisserman, "Very deep convolutional networks for large-scale image recognition", arXiv preprint arXiv:1409.1556 (2014).

[21] Jakimovski, Goran, and Danco Davcev, "Lung cancer medical image recognition using Deep Neural Networks", In 2018 Thirteenth International Conference on Digital Information Management (ICDIM), pp. 1-5. IEEE, 2018.

[22] Zhou, Zongwei, Md Mahfuzur Rahman Siddiquee, Nima Tajbakhsh, and Jianming Liang,"Unet++: A nested u-net architecture for medical image segmentation", In Deep Learning in Medical Image Analysis and Multimodal Learning for Clinical Decision Support, pp. 3-11. Springer, Cham, 2018.

[23] Tekade, R. and Rajeswari, K,” Lung cancer detection and classification using deep learning”, In 2018 Fourth International Conference on Computing Communication Control and Automation (ICCUBEA) (pp. 1-5). IEEE, 2018, August.

[24] Pehrson, Lea Marie, Michael Bachmann Nielsen, and Carsten Ammitzbøl Lauridsen. "Automatic pulmonary nodule detection applying deep learning or machine learning algorithms to the LIDC-IDRI database: a systematic review." Diagnostics 9, no. 1 (2019): 29.

[25] W. Rahane, H. Dalvi, Y. Magar, A. Kalane and S. Jondhale, "Lung Cancer Detection Using Image Processing and Machine Learning HealthCare," 2018 International Conference on Current Trends towards Converging Technologies (ICCTCT), Coimbatore, 2018, pp. 1-5, doi: 10.1109/ICCTCT.2018.8551008.

[26] Y. F. Riti, H. A. Nugroho, S. Wibirama, B. Windarta and L. Choridah, "Feature extraction for lesion margin characteristic classification from CT Scan lungs image," 2016 1st International Conference on Information Technology, Information Systems and Electrical Engineering (ICITISEE), Yogyakarta, 2016, pp. 54-58, doi: 10.1109/ICITISEE.2016.7803047.

[27] Li, Yu Ting and Jiun In Guo. "A VGG-16 Based Faster RCNN Model for PCB Error Inspection in Industrial AOI Applications.” 2018 IEEE International Conference on Consumer Electronics-Taiwan (ICCE-TW) (2018): 1-2.

[28] Zhou, Zongwei \& Rahman Siddiquee, Md Mahfuzur \& Tajbakhsh, Nima \& Liang, Jianming,"UNet++: A Nested U-Net Architecture for Medical Image Segmentation", 4th Deep Learning in Medical Image Analysis (DLMIA) Workshop,Spain,2018. 